\title{
Glycopeptide antibiotic biosynthesis
}

\author{
Grace Yim, Maulik N Thaker, Kalinka Koteva and Gerard Wright
}

Glycopeptides such as vancomycin, teicoplanin and telavancin are essential for treating infections caused by Gram-positive bacteria. Unfortunately, the dwindled pipeline of new antibiotics into the market and the emergence of glycopeptide-resistant enterococci and other resistant bacteria are increasingly making effective antibiotic treatment difficult. We have now learned a great deal about how bacteria produce antibiotics. This information can be exploited to develop the next generation of antimicrobials. The biosynthesis of glycopeptides via nonribosomal peptide assembly and unusual amino acid synthesis, crosslinking and tailoring enzymes gives rise to intricate chemical structures that target the bacterial cell wall. This review seeks to describe recent advances in our understanding of both biosynthesis and resistance of these important antibiotics. The Journal of Antibiotics (2014) 67, 31-41; doi:10.1038/ja.2013.117; published online 13 November 2013

Keywords: biosynthesis; glycopeptide; resistance; teicoplanin; vancomycin

\section{INTRODUCTION}

The glycopeptide antibiotics (GPAs) are essential for the control of infectious disease caused by Gram-positive pathogenic bacteria. Three GPAs are in current clinical use (Figure 1 and Table 1): the natural products vancomycin and teicoplanin, and the semisynthetic drug telavancin (Vibativ) approved in 2009 by the Food and Drug Administration (FDA). The GPAs are remarkable in their chemical structures, consisting of highly crosslinked and diversely modified core heptapeptides, their biosynthesis via nonribosomal peptide assembly and unusual amino acid synthesis, their antibiotic activity through binding to peptidoglycan and in the associated resistance mechanisms that act via complex alteration of cell wall synthesis.

Vancomycin (from the root word 'vanquish') was discovered in 1953, isolated from a strain of Amycolatopsis orientalis found in a soil sample collected in Borneo. ${ }^{1}$ It was introduced into the clinic 5 years later by the company Eli Lilly and has been a mainstay for the treatment of serious infections caused by Gram-positive pathogens ever since. Initially, vancomycin was used only sparingly given the effectiveness and favorable pharmacology of other antibiotics such as semisynthetic $\beta$-lactams and because of adverse side effects due to contaminants in vancomycin preparations owing to the challenge in obtaining the drug in a highly purified form. However, improvements in drug purification along with the increased prevalence of methicillin-resistant Staphylococcus aureus in the late 1970s resulted in a commensurate rise in the use and dependence on vancomycin. ${ }^{2}$ In Europe, the lipoglycopeptide antibiotic (LGPA) teicoplanin A2 purified from Actinoplanes teichomyceticus was also introduced for clinical use in the mid-1980s. Teicoplanin showed antibiotic activity comparable in spectrum to vancomycin but with superior activity toward enterococci and other organisms. ${ }^{3,4}$
GPAs act by binding to the N-acyl-D-Ala-D-Ala termini of peptidoglycan and its precursor Lipid II (Figure 2). This binding effectively sequesters the substrate for two key enzymes critical to cell wall synthesis: the transglycosylases that transfer the $N$-acetyl-muramic acid- $N$-acetyl-glucosamine pentapeptide subunits from Lipid II to the anchored cell wall, and the D,D-transpeptidases that crosslink strands of peptidoglycan. The result is an inability to grow and rigidify the cell wall that blocks cell division and weakens the wall, resulting in cell death (Figure 3 ). This mode of action that targets the biochemically complex and ubiquitous cell wall on the outside of Gram-positive bacteria is an ingenious strategy to avoid the rapid emergence of resistance through upregulation of pumps, downregulation of transport proteins or mutations in target enzymes, effectively eliminating three common mechanisms of antibiotic resistance.

The emergence of enterococci resistant to vancomycin (VRE) in the late 1980s therefore came as a surprise to the clinical community that increasingly relied on GPAs to cure infectious disease. The global spread of VRE since then has triggered an intense effort to understand the biochemistry and genetics of resistance and the exploration of GPA biosynthesis with a goal of overcoming resistance. We have now learned a great deal about how producing bacteria assemble GPAs and LGPAs and how we may exploit this information in the development of next-generation antibiotics that avoid resistance. This review seeks to describe recent advances in our understanding of both biosynthesis and resistance of these important antibiotics.

\section{GPA BIOSYNTHETIC CLUSTERS}

Almost a dozen GPA biosynthetic clusters have been sequenced, annotated and characterized in the past 15 years. Half of which were published in the last few years. Sequencing of cosmid clones or 
Teicoplanin (T) type scaffold

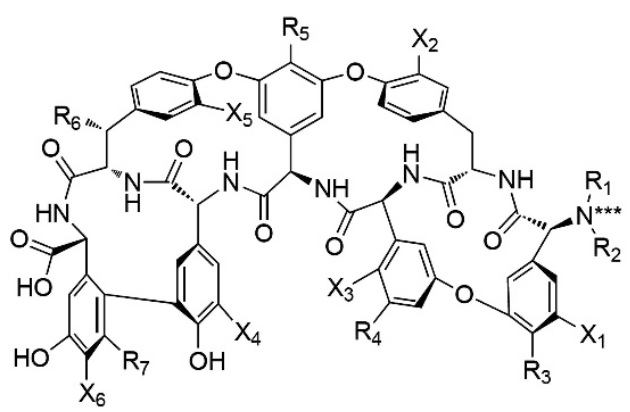

Vancomycin (V) type scaffold

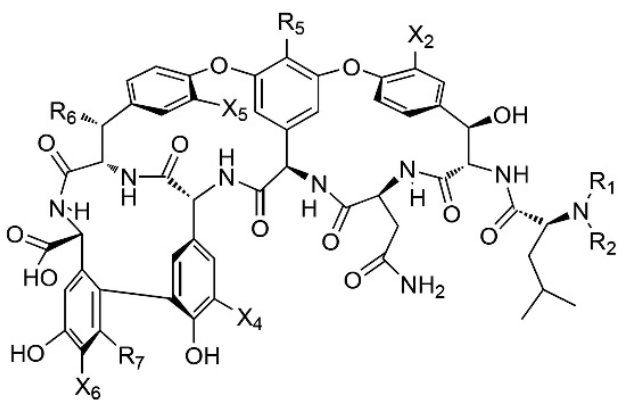

R5 Groups<smiles>CCCCC=CCCC(=O)N[C@H](OCC)C(O)(O)C(O)OCC</smiles>

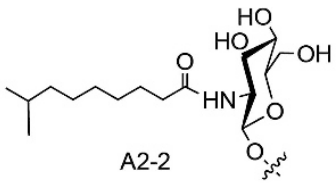

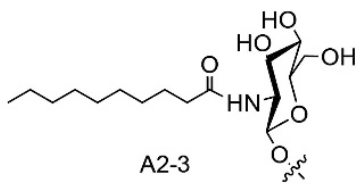<smiles>CCC(C)CCCCCCC(=O)NCC(O)COC(O)C(O)(O)O</smiles><smiles>CCOC(NC(=O)CCCCCCCC(C)C)OCC(O)(O)C(O)(O)O</smiles><smiles>CCCCCCCCCC(=O)NC(O)C(O)C(O)C(O)C(=O)O</smiles>

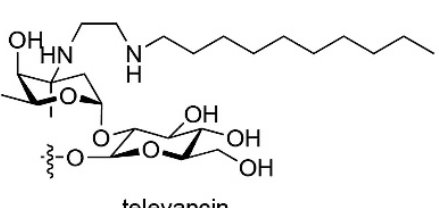





televancin L-vancosamine-1,2-glucose

L-4-epi-vancosamine-1,2-glucose<smiles>COC(OC)C1OCC(O)C(O)C1O</smiles>

2-O-methyl-glucose<smiles>OC1COC(O)C(O)C(O)C1O</smiles>

D-glucose

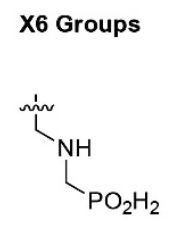

televancin
R6 Groups

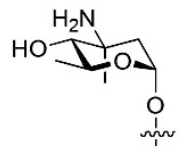

L-4-epi-vancosamine

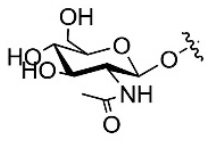

$\mathrm{N}$-acetyl-glucosamine

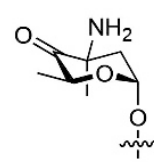

dehydrovancosamine
R7 Groups



D-mannose

Figure 1 Glycopeptide $\mathrm{R}$ and $\mathrm{X}$ groups described in Table 1 .

whole-genome sequencing methods have allowed the annotation of the balhimycin (bal), ${ }^{5}$ chloroeremomycin (cep) ${ }^{6}$ vancomycin $(v p s),{ }^{7,8}$ A40926 (dbv), ${ }^{9}$ A47934 (sta),${ }^{10}$ teicoplanin $($ tcp/tei $),{ }^{11,12}$ pekiskomycin $(p e k)^{13}$ and UK-68597 $(a u k)^{14}$ biosynthetic clusters. The $d b v$ designation of A40926 is named after its clinically relevant semisynthetic derivative dalbavancin. Two designations exist for the teicoplanin clusters since two manuscripts describing the teicoplanin biosynthetic cluster with different ORF numberings were published in the same year. ${ }^{11,12}$ Assembly of sequences from metagenomic samples ${ }^{15,16}$ have allowed the identification of the following clusters: VEG (vancomycin-like environmental DNA derived gene cluster), TEG (teicoplanin-like environmental DNA derived gene cluster), CA37, CA878, CA915 and two teicoplanin-type molecules with the eSNaPD identification numbers 15 and $26 .{ }^{15-17}$

\section{HEPTAPEPTIDE SCAFFOLDS}

Antimicrobial GPAs are heptapeptides synthesized by nonribosomal peptide synthetases (NRPS). NRPS complexes are multimodular enzyme machines that assemble amino acids in an assembly line manner. One module corresponds to one amino acid; the primary sequence of the peptide product is dictated by the order of the modules in the NRPS. ${ }^{18}$

Each NRPS module can encode various domains. ${ }^{18,19}$ This includes but is not limited to condensation $(\mathrm{C})$, adenylation (A), thiolation ( $\mathrm{T}$, also known as peptidyl carrier protein $(\mathrm{PCP})$ ), epimerization (E) and thioesterase (Te) domains. ${ }^{11,12}$ The $\mathrm{C}, \mathrm{A}$ and $\mathrm{T}$ domains are essential in each module, whereas a single Te domain terminates synthesis and cleaves the peptide chain from the NRPS assembly. Adenylation domains are responsible for substrate recognition and 
Table 1 Glycopeptide producers and substituents

\begin{tabular}{|c|c|c|c|c|c|c|c|c|c|c|c|c|c|c|c|c|}
\hline GPA & Producereference & Scaffold & $x_{1}$ & $x_{2}$ & $x_{3}$ & $x_{4}$ & $x_{5}$ & $x_{6}$ & $R_{1}$ & $R_{2}$ & $R_{3}$ & $R_{4}$ & $R_{5}$ & $R_{6}$ & $R_{7}$ & $\begin{array}{l}\text { Scaffold } \\
\text { differences }\end{array}$ \\
\hline A40926 & $\begin{array}{l}\text { Nonomuraea sp. } \\
\text { ATCC } 39727^{9}\end{array}$ & $\mathrm{~T}$ & $\mathrm{H}$ & $\mathrm{H}$ & $\mathrm{Cl}$ & $\mathrm{H}$ & $\mathrm{Cl}$ & $\mathrm{H}$ & $\mathrm{CH}_{3}$ & $\mathrm{H}$ & $\mathrm{OH}$ & $\mathrm{OH}$ & As shown & $\mathrm{OH}$ & D-mannose & None \\
\hline A47934 & $\begin{array}{l}\text { Streptomyces } \\
\text { toyocaensis } \\
\text { NRRL } 15009^{10}\end{array}$ & $\mathrm{~T}$ & $\mathrm{H}$ & $\mathrm{Cl}$ & $\mathrm{H}$ & $\mathrm{Cl}$ & $\mathrm{Cl}$ & $\mathrm{H}$ & $\mathrm{H}$ & $\mathrm{H}$ & $\mathrm{SO}_{4}^{-}$ & $\mathrm{OH}$ & $\mathrm{OH}$ & $\mathrm{OH}$ & $\mathrm{OH}$ & None \\
\hline CA878 & Metagenomic ${ }^{15}$ & $\mathrm{~T}$ & $?^{a}$ & $?^{\mathrm{a}}$ & $?^{\mathrm{a}}$ & $?^{\mathrm{a}}$ & $?^{\mathrm{a}}$ & $?^{\mathrm{a}}$ & $?^{\mathrm{a}}$ & $?^{\mathrm{a}}$ & $?^{a}$ & $?^{a}$ & $?^{a}$ & $?^{a}$ & $?^{\mathrm{a}}$ & Tyr2-> Bht2 \\
\hline CA915 & Metagenomic ${ }^{15}$ & $\mathrm{~T}$ & $?^{\mathrm{a}}$ & $?^{a}$ & $?^{\mathrm{a}}$ & $?^{a}$ & $?^{\mathrm{a}}$ & $?^{\mathrm{a}}$ & $?^{a}$ & $?^{\mathrm{a}}$ & $?^{\mathrm{a}}$ & $?^{a}$ & $?^{a}$ & $?^{a}$ & $?^{a}$ & $\begin{array}{l}\text { Dpg3-> Hpg3 } \\
\text { Bht6-> Tyr6 }\end{array}$ \\
\hline $\begin{array}{l}\text { eSNaPD id } \\
15 \text { and } 26\end{array}$ & Metagenomic ${ }^{17}$ & $\mathrm{~T}$ & $?^{a}$ & $?^{a}$ & $?^{a}$ & $?^{a}$ & $?^{a}$ & $?^{a}$ & $?^{a}$ & $?^{a}$ & $?^{a}$ & $?^{a}$ & $?^{a}$ & $?^{a}$ & $?^{\mathrm{a}}$ & None $^{a}$ \\
\hline TEG & Metagenomic ${ }^{16}$ & $\mathrm{~T}$ & $?^{a}$ & $?^{a}$ & $?^{a}$ & $?^{a}$ & $?^{\mathrm{a}}$ & $?^{a}$ & $?^{\mathrm{a}}$ & $?$ & $?^{\mathrm{a}}$ & $\mathrm{SO}_{4}^{-}$ & $\mathrm{SO}_{4}^{-}$ & $\mathrm{SO}_{4}^{-}$ & $?^{a}$ & Tyr2->Bht2 \\
\hline UK-68597 & $\begin{array}{l}\text { Acintoplanes sp. } \\
\text { ATCC } 53533^{14}\end{array}$ & $\mathrm{~T}$ & $\mathrm{Cl}$ & $\mathrm{Cl}$ & $\mathrm{H}$ & $\mathrm{Cl}$ & $\mathrm{Cl}$ & $\mathrm{H}$ & - & - & $\mathrm{OH}$ & $\mathrm{SO}_{4}^{-}$ & $\begin{array}{l}\text { L-vancosa- } \\
\text { mine-1,2- } \\
\text { glucose }\end{array}$ & $\mathrm{OH}$ & $\mathrm{OH}$ & $\begin{array}{l}* * * \text { Terminal } \\
\mathrm{NH}_{2} \text { replaced } \\
\text { by }=0\end{array}$ \\
\hline VEG & Metagenomic ${ }^{16}$ & $\mathrm{~T}^{\mathrm{b}}$ & $\mathrm{H}^{\mathrm{a}}$ & $\mathrm{Cl}^{\mathrm{a}}$ & $\mathrm{H}^{\mathrm{a}}$ & $\mathrm{H}^{\mathrm{a}}$ & $\mathrm{Cl}^{\mathrm{a}}$ & $\mathrm{H}^{\mathrm{a}}$ & $\mathrm{CH}_{3}{ }^{\mathrm{a}}$ & $\mathrm{CH}_{3}{ }^{\mathrm{a}}$ & $?^{\mathrm{a}}$ & $?^{\mathrm{a}}$ & $?^{a}$ & $?^{a}$ & $?^{\mathrm{a}}$ & $\begin{array}{l}\text { Tyr2->Bht2 } \\
\text { Dpg3-> Hpg3 }\end{array}$ \\
\hline Balhimycin & $\begin{array}{l}\text { Amycolatopsis } \\
\text { balhimycina }{ }^{5}\end{array}$ & V & - & $\mathrm{Cl}$ & - & $\mathrm{H}$ & $\mathrm{Cl}$ & $\mathrm{H}$ & $\mathrm{CH}_{3}$ & $\mathrm{H}$ & - & - & D-glucose & $\begin{array}{l}\text { Dehydro- } \\
\text { vancosamine }\end{array}$ & $\mathrm{OH}$ & None \\
\hline Chloroeremomycin & $\begin{array}{l}\text { Amycolatopsis } \\
\text { orientalis }^{6}\end{array}$ & V & - & $\mathrm{Cl}$ & - & $\mathrm{H}$ & $\mathrm{Cl}$ & $\mathrm{H}$ & $\mathrm{CH}_{3}$ & $\mathrm{H}$ & - & - & $\begin{array}{l}\text { L-4-epi-vanco- } \\
\text { samine-1,2- } \\
\text { glucose }\end{array}$ & $\begin{array}{l}\text { - L-4-epi- } \\
\text { vancosamine }\end{array}$ & $\mathrm{OH}$ & None \\
\hline
\end{tabular}

Abbreviations: GPA, glycopeptide antibiotic; TEG, teicoplanin-like environmental DNA derived gene cluster; VEG, vancomycin-like environmental DNA derived gene cluster. aproposed or unknown modifications from full or partial annotation of the biosynthetic cluster; GPAs were not isolated, only stf genes were characterized. ${ }^{16}$

bThis cluster has a predicted crosslinking that is a hybrid between teicoplanin and vancomycin patterns. '**** Refers to the same symbols indicated in Figure 1.

activation and consequently their sequence is used to predict the amino acid encoded by the corresponding module. ${ }^{20}$ All intermediates of the NRPS assembly line are covalently bound as thioesters to the 4-phosphopantetheine arm of the PCP. ${ }^{18}$ Condensation domains catalyze peptide bond formation between two adjacent PCP-bound intermediates. ${ }^{18}$ Epimerase domains convert the stereochemistry of amino acids from L- to a mixture of D- and Lby deprotonation and reprotonation of the amino acid $\alpha$-carbon when it is tethered to a PCP domain. ${ }^{21,22}$ The downstream condensation domain is specific for one stereoisomer. ${ }^{23}$

The various GPAs have several different module arrangements within the NRPS ORFs. In the cases where module arrangements were not published in the original manuscript of the respective biosynthetic cluster, arrangements were predicted using antiSMASH. ${ }^{24}$ The cep, ${ }^{6}$ bal, ${ }^{5} v p s,{ }^{7,8}$ pek $^{13}$ and eSNaPD $26^{17}$ biosynthetic clusters have a $3 / 3 / 1$ arrangement in which the first NRPS ORF has three modules encoding three amino acids, the second NRPS ORF has three modules encoding three amino acids and the third NRPS ORF has one module encoding the last amino acid (Figure 4a). The $\mathrm{X}$ domain ${ }^{9,11,17,25}$ is presumed to be a nonfunctional $\mathrm{E}$ or $\mathrm{C}$ domain. The $s t a,{ }^{10} a u k^{14}$ and CA $37^{16}$ clusters have a 2/1/3/1 arrangement and an extra $\mathrm{X}$ domain at the end of module 3 (Figure $4 \mathrm{~b}$ ). The teicoplanin,,${ }^{11,12}$ A40926, ${ }^{9}$ TEG, ${ }^{16}$ VEG,${ }^{16}$ CA898 ${ }^{16}$ and eSNaPD $15^{17}$ clusters are similar to the module arrangement of sta/auk except that module 3 has no X domain (Figure 4c). The predicted arrangement of one of the biosynthetic cluster from a soil metagenomic sample, CA915, ${ }^{15}$ is particularly unique with a $1 / 1 / 1 / 3 / 1$ arrangement: module 1 (A-T-C), module 2 (A-T-E), module 3 (C-A-T), module 4-6 (C-A-T-E C-A-T-E-C-A-T) and module 7 (C-A-T-X-TE).

The two main antimicrobial GPA scaffolds are the vancomycin and teicoplanin types (Figure 4d). Like many natural products, GPAs contain an assortment of proteinogenic and nonproteinogenic amino acids. The proteinogenic amino acids described include Tyr, Leu, Asn, $\mathrm{Ala}$ and Glu. The nonproteinogenic amino acids incorporated into GPAs are 4-hydroxyphenylglycine (Hpg), 3,5-dihydroxyphenylglycine (Dpg) and $\beta$-hydroxytyrosine (Bht). 




Figure 2 A schematic of the interactions between vancomycin and Lipid II. H-bonds (dashed lines) are between the backbone of vancomycin and the D-AlaD-Ala terminus of Lipid II.
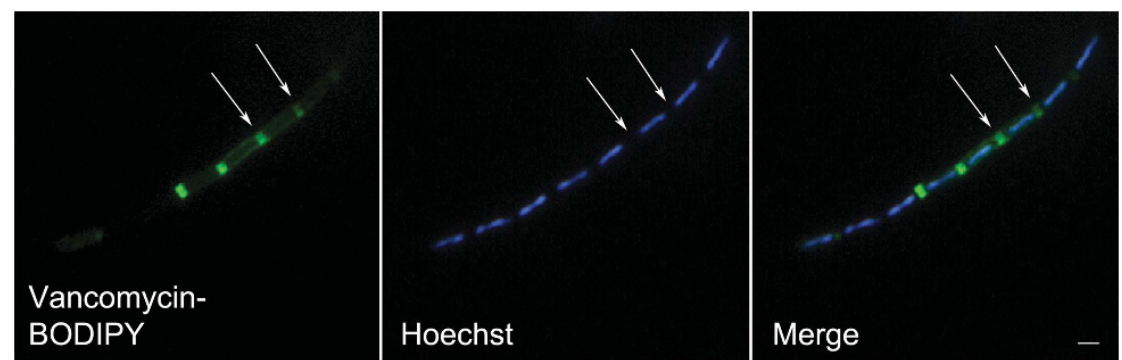

Figure 3 Localization of vancomycin-BODIPY in Bacillus subtilis cells. Cells were doubly stained with vancomycin-BODIPY (vancomycin labeled with the fluorophore BODIPY on its free amine group) and the DNA stain Hoechst. Fluorescence microscopy reveals regions densely stained with vancomycin-BODIPY (arrows) presumably corresponding to the septum. BODIPY is 4,4-difluoro-4-bora-3a,4a-diaza-s-indacene. Scale bar, $2 \mu \mathrm{m}$.

Many of the recently described GPAs have heptapeptide sequences that differ from the vancomycin- and teicoplanin-type sequences (Table 1) and many more GPAs with variable sequences exist. ${ }^{26}$ One recent example is an unusual GPA pekiskomycin (Figure $4 \mathrm{~d}$ and e) whose producer was isolated using a resistance guided isolation method. ${ }^{13}$

\section{OXIDATIVE CROSSLINKING}

The number of crosslinks and the number of corresponding P450type monooxygenases differ in the two types of GPAs. Vancomycintype clusters have three oxygenases, OxyA, OxyB and OxyC, responsible for crosslinking, whereas teicoplanin-type clusters have an additional enzyme, OxyE. Examination of balhimycin precursors in the production media of Amycolatopsis balhimycina oxy mutants were consistent with an $\mathrm{OxyB}>\mathrm{OxyA}>\mathrm{OxyC}^{27}$ order of crosslinking. OxyB and OxyA form diaryl ethers (C-O-C bonds) between Hpg4/ Bht6 and Bht2/Hpg4 (numbers indicate the position of the respective amino acid from the $\mathrm{N}$-terminus of the peptide), respectively, whereas OxyC mediates the C-C bond formation between Hpg5 and Dpg7. ${ }^{27}$ Complementation of the mutants with the homologous vancomycin biosynthetic genes suggested that the proteins were functionally equivalent. ${ }^{27}$ Similar genetic and chemical analyses with the A47934 (sta) monooxygenases ${ }^{28}$ and crystal structures of the teicoplanin $\mathrm{OxyE}^{29,30}$ suggested that StaG and StaF have some substrate flexibility but that the preferred order of crosslinking is StaH (OxyB), StaG (OxyE), StaF (OxyA) and StaJ (OxyC) where OxyE mediates the 

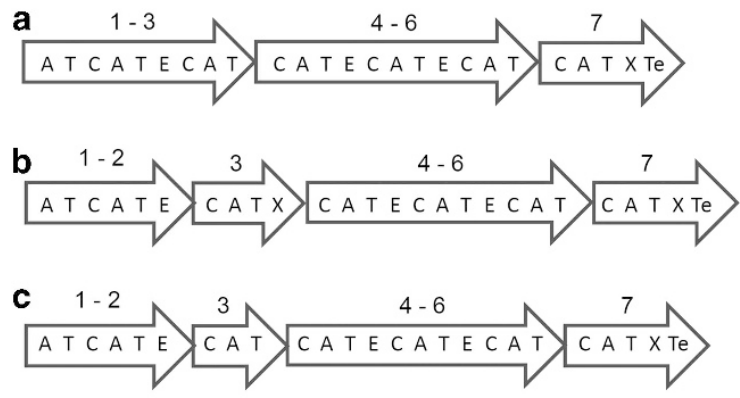

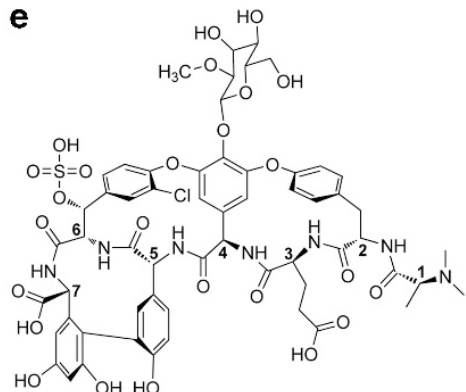

d Heptapeptide amino acid sequences

$\begin{array}{lcccccccccc}\text { Amino acid no. } & \text { N - } & \mathbf{1} & \mathbf{2} & \mathbf{3} & \mathbf{4} & \mathbf{5} & \mathbf{6} & \mathbf{7} & \mathbf{- C} \\ & & & & & & & & & \\ \text { Vancomycin type } & \mathrm{N}- & \text { Leu } & \text { Bht } & \text { Asn } & \text { Hpg } & \text { Hpg } & \text { Bht } & \text { Dpg } & \text { - C } \\ \text { Teicoplanin type } & \text { N - } & \text { Hpg } & \text { Tyr } & \text { Dpg } & \text { Hpg } & \text { Hpg } & \text { Bht } & \text { Dpg } & - \text { C } \\ \text { Pekiskomycin } & \text { N - } & \text { Ala } & \text { Tyr } & \text { Glu } & \text { Hpg } & \text { Hpg } & \text { Bht } & \text { Dpg } & - \text { C }\end{array}$

Figure 4 (a-c) Module and domain arrangements with the nonribosomal peptide synthetase (NRPS) genes of the various glycopeptide biosynthetic clusters. Numbers indicate the respective modules. (a) A 3/3/1 module arrangement is shown. (b, c) Two different 2/1/3/1 arrangements are shown. (d) The amino acid sequence of the various types of glycopeptides from the $\mathrm{N}$ - to C-terminus of the peptide. Shaded areas highlight sequence differences. (e) The structure of pekiskomycin.

diaryl ether formation between $\mathrm{Hpg} 1$ and Dpg3 $3{ }^{29}$ In vitro studies with vancomycin $\mathrm{OxyB}$ suggested that the preferred substrate is the linear peptide tethered to a PCP domain rather than the free peptide. ${ }^{31}$ This suggested that oxidative crosslinking occurs while the peptide is covalently bound to a PCP domain of the NRPS. ${ }^{31}$

\section{NON-PROTEINOGENIC AMINO ACIDS}

Genes encoding the biosynthetic enzymes of the non-proteinogenic amino acids Dpg, Hpg and Bht are usually located within the biosynthetic cluster. The enzymes required for Dpg biosynthesis have been identified and characterized in vitro. ${ }^{32,33}$ Five genes, $d p g A-D$ and $h p g T$ are required for Dpg biosynthesis from malonyl-CoA ${ }^{32}$ (Figure 5a). DpgA, B and D convert four malonyl-CoA molecules to 3,5-dihydroxyphenylacetyl-CoA and three free $\mathrm{CoASH}$ derivatives. $^{32}$ Action of DpgC then catalyzes 3,5-dihydroxyphenylglyoxylate synthesis. ${ }^{32} \mathrm{~A}$ deletion mutant of the balhimycin producer and in vitro characterization showed that the final conversion to Dpg is then mediated by the transaminase HpgT (also known as Pgat). ${ }^{33,34}$

The proposed pathway for Hpg biosynthesis involves four enzymes $^{35}$ (Figure $5 \mathrm{~b}$ ). The pathway begins with conversion of the common metabolite prephenate to p-hydroxyphenylpyruvate by prephenate dehydrogenase (Pdh). ${ }^{35}$ In vitro characterization of 4-hydroxymandelate synthase (HmaS), hydroxymandelate oxidase (Hmo) and p-hydroxyphenylglycine transaminase (HpgT/Pgat), respectively, catalyzed the transformations from p-hydroxyphenylpyruvate to L-p-hydroxymandelate to p-hydroxylbenzoylformate to Hpg. ${ }^{35}$

Bht biosynthesis in vancomycin-type GPAs differs from the pathway in teicoplanin-type producers. Enzymes involved in $\beta$-hydroxylation of nonribosomally encoded amino acids were first described in the novobiocin and nikkomycin producers. ${ }^{36,37}$ Similar enzymes are present in vancomycin-type producers and are responsible for Bht synthesis from Tyr: BpsD, OxyD and $\mathrm{Bhp}^{38}$ (Figure $5 \mathrm{c}$ ). Gene deletions of $b p s D, \quad 0 x y D$ or $b h p$ abolished GPA biosynthesis in A. balhimycina. ${ }^{39-41} \mathrm{BpsD}$ (orf19 in cep) is a minimal NRPS module that lacks a C domain but contains a PCP domain and an A domain that presumably activates Tyr. ${ }^{40}$ In vitro binding assays with
OxyD bal and structural data suggest that $\mathrm{OxyD}$ does not hydroxylate free Tyr but rather Tyr covalently bound to the PCP domain of BpsD. ${ }^{42}$ Similarly, exogenous Bht supplementation of oxyD mutants was able to restore synthesis of balhimycin, suggesting that the bal A domains in modules 2 and 6 recognize Bht (rather than Tyr). ${ }^{39}$ Subsequent in vitro analysis of the balhimycin protein Bhp (orf18 in сер) using a Bht-S-PCP surrogate, S- $\beta$-hydroxytyrosyl- $N$-acetyl cysteamine thioester, as a substrate showed hydrolysis resulting in release of free Bht. ${ }^{43}$ This identified Bhp as a thioesterase responsible for the release of Bht from the PCP domain of BpsD..$^{43}$

In the teicoplanin-type producer of A40926, inactivation of a putative hydroxylase Orf28 resulted in A40926 with Tyr in place of Bht6. ${ }^{44}$ Unlike balhimycin, feeding experiments with Bht in an orf28deficient mutant did not restore production of A40926, implying that in teicoplanin-type producers, hydroxylation occurs after Tyr has been incorporated into the peptide chain by the NRPS. ${ }^{44}$ Homologs in the teicoplanin-type producers of teicoplanin, A47934 and UK-68597 are Tcp $25,{ }^{11}$ StaM $^{10}$ and Auk24, ${ }^{14}$ respectively.

\section{TAILORING ENZYMES}

GPA tailoring enzymes such as glycosyltransferases (Gtfs), acyltransferases (Atfs), halogenases, sulfotransferases (Stfs) and methyltransferases (Mtfs) modify specific residues on the heptapeptide backbones. Tailoring of scaffolds can alter chemical and biological properties including solubility, dimerization, resistance induction, antimicrobial activity, etc. Importantly, similar enzymes appear to maintain regiospecificity regardless of the GPA scaffold. ${ }^{45}$

\section{GLYCOSYLATION}

Glycosylation is a frequently occurring GPA modification. An exception is A47934, a teicoplanin-type GPA aglycone produced by Streptomyces toyocaensis. ${ }^{10}$ As with the other tailoring enzymes, glycosylation reactions are regiospecific and modify one specific amino acid on the heptapeptide scaffold. Glycosylation is dominantly directed to amino acid 4 and less frequently at positions 5 and 6 .

The original in vitro characterizations of GPA Gtfs were conducted with GtfA, GtfB and GtfC from the chloroeremomycin biosynthetic 




Figure 5 Proposed schemes for 3,5-dihydroxyphenylglycine (Dpg), 4-hydroxyphenylglycine (Hpg), $\beta$-hydroxytyrosine (Bht) and 4-epi-vancosamine biosynthesis. Simplified biosynthesis schemes for the nonproteinogenic amino acids (a) Dpg, (b) Hpg, (c) Bht in the producer of balhimycin (vancomycintype) and (d) L-4-epi-vancosamine.

cluster and GtfD and GtfE from the vancomycin biosynthetic cluster. $^{46,47}$ Subsequent Gtf annotation for the teicoplanin, A40926 and balhimycin biosynthetic clusters has followed the A, B, C nomenclature and presumably glycosylate with similar regiospecificity. GtfB and GtfE utilize TDP-glucose to add a single glucose to Hpg4 of the heptapeptide; subsequently, GtfC and GtfD attach a secondary sugar moiety, 4-epi-vancosamine and vancosamine, respectively, to glucose resulting in a disaccharide ${ }^{46,48,49}$ (Figure 1 depicts mono- and disaccharide structures).

In different clusters, GtfAs add unique monosaccharides to amino acid 6: epi-vancosamine in cep, ${ }^{49}$ dehydrovancosamine in $b a l^{5}$ and $\mathrm{N}$-acetyl-glucosamine in $t c p / t e i .^{11,12}$ The relative efficiency of a given Gtf in vivo appears to result in a constellation of related GPAs being produced by a given strain with a variety of glycosylation patterns. For example, in the balhimycin biosynthetic cluster, there are three Gtfs but only two glycosylations in the major product balhimycin. The third Gtf is proposed to glycosylate minor products of biosynthesis, balhimycin $\mathrm{V}$ and dechlorobalhimycin $\mathrm{V}$ that have three glycosylations. ${ }^{5}$

The genes involved in the biosynthesis of vancosamine and the related sugars usually exist within the GPA biosynthetic cluster. ${ }^{38}$ The enzymes in the 4-epi-vancosamine biosynthesis pathway of chloroeremomycin (EvaA, B, C, D and E) have been reconstituted in vitro. ${ }^{50}$ TDP-4-keto-6-deoxy-D-glucose is converted to TDPepi-vancosamine in a process that involves $\mathrm{C}-2$ deoxygenation, $\mathrm{C}-3$ amination, C-3 methylation, C-5 epimerization and C-4 ketoreduction by EvaA-E, respectively ${ }^{50}$ (Figure $5 \mathrm{~d}$ ). Balhimycin has a dehydrovancosamine at Bht6 rather than a 4-epi-vancosamine. Its biosynthetic cluster contains EvaA-D but EvaE exists as a pseudogene. ${ }^{38}$ This is consistent with the expected product of EvaA-D, dehydrovancosamine, which lacks a C-4 ketoreduction. ${ }^{38,50}$

Both A40926 and teicoplanin have mannose at Dpg7. The putative mannosyltransferases $d b v 20$ and $t c p 15$ have been identified by their similarity to another NRPS mannosyltransferase involved in ramoplanin biosynthesis. ${ }^{9,11}$ These proteins look distinct from the other Gtfs from GPA biosynthetic clusters that use NDP sugars as substrates. ${ }^{9}$

\section{ACYLATION}

Acylation is a modification present in the naturally occurring LGPAs teicoplanin and A40926 and some semisynthetic derivatives designed for clinical use such as telavancin (Figure 1 and Table 1). Acylation has proven to impart favorable antibacterial characteristics and activity against some VRE strains. ${ }^{51}$ In teicoplanin and A40926, the Atfs add an acyl group to the 2-aminoglucosylated Hpg4 of the heptapeptide scaffold. ${ }^{52}$ In clinical preparations of teicoplanin, the 
substrate promiscuity of the Atf results in a family of compounds being produced: five major teicoplanins (A2-1 to A2-5) and sometimes four minor components that all have slight differences in the acyl chain. ${ }^{12}$ Overexpression and isolation of the teicoplanin and A40926 Atfs (tAtf and aAtf) tested against a variety of acyl-CoA substrates showed that the longer acyl chains $\left(\mathrm{C}_{10}\right.$ and $\left.\mathrm{C}_{12}\right)$ were the best substrates for both enzymes, consistent with a $N$-decanoyl modification in the dominant products of both biosynthetic clusters. ${ }^{52}$ Interestingly, both tAtf and aAtf show a high degree of promiscuity in substrate recognition as well as regiospecificity. Both enzymes were able to accept 2-aminoglucosyl derivatives of teicoplanin and vancomycin aglycones with similar efficiencies. ${ }^{52}$ Surprisingly, tAtf and aAtf showed promiscuous regiospecificity at high $\mathrm{pH}$, acylating the 6-N-aminoglucosyl-teicoplanin aglycone at the amino group and glucosyl-teicoplanin aglycone at a hydroxyl group but with a $k_{\text {cat }}$ three orders of magnitude lower than for the 2-amino group. ${ }^{52}$

Although tGtfB is relatively promiscuous in terms of the sugar donor that it utilizes, UDP- $N$-acetyl-glucosamine (UDP-GlcNAc) is most likely the biological sugar donor in GPA producers. ${ }^{53}$ tGtfB has been shown to utilize UDP-GlcNAc, UDP-2-aminoglucose and UDP$\left(N\right.$-acyl)-2-aminoglucose with similar catalytic efficiencies, ${ }^{53}$ but a deacetylase has been identified in the teicoplanin and A40926 biosynthetic clusters, Orf $2 * 12$ and Dbv21, ${ }^{9}$ respectively. ${ }^{54}$ In vitro experiments with both proteins suggested that teicoplanin aglycone modified with GlcNAc at $\mathrm{Hpg} 4$ was the substrate of the deacetylases with no activity observed against UDP-GlcNAc (free sugar) or teicoplanin aglycone modified with GlcNAc at Bht6. ${ }^{54}$ Rather than UDP-2-aminoglucose or UDP-( $N$-acyl)-2-aminoglucose, biologically, it seems most rational to assume that GlcNAc is the sugar utilized during the glycosylation reactions of LGPAs given that the concentration of UDP-GlcNAc in the cell is likely much higher relative to the other glycosyl donors ${ }^{53}$ and that teicoplanin also has a GlcNAc modification at Bht6 (Figure 1 and Table 1).

The order of acylation and glycosylation in teicoplanin was determined using a series of systematic in vitro experiments using overexpressed Atf, tGtfA and tGtfB with teicoplanin in various stages of acylation and glycosylation as substrates. ${ }^{53}$ The conclusions from these experiments are summarized in the 'Timing of biosynthetic events' section.

\section{CHLORINATION}

Chlorination is a common GPA and LGPA modification that is not well understood, although it is hypothesized to improve GPA dimerization $^{55}$ that may in turn positively enhance antimicrobial activity. ${ }^{56}$ Genetic experiments have identified a flavin-dependent halogenase within the balhimycin biosynthetic cluster. ${ }^{41}$ Inactivation of bhaA resulted in isolation of dechlorobalhimycin from fermentations of A. balhimycina, supporting the hypothesis that one enzyme is responsible for chlorination of both Bhts. ${ }^{41}$ Unlike Bht supplementation, when growth of an A. balhimycina bpsD mutant (unable to synthesize Bht) is supplemented with chloro-Bht, balhimycin synthesis is not restored, suggesting that chlorination occurs after Bht is incorporated into the nascent peptide chain. ${ }^{39}$

Other GPA biosynthetic clusters have one to two similar halogenases within their clusters ${ }^{14,38}$ corresponding to one to four chlorines in their structure (Figure 1 and Table 1). Clusters with one halogenase are commonly chlorinated at Bht6 and Tyr/Bht2, whereas the clusters with two halogenases are additionally halogenated at Hpg1, Dpg3 or Hpg5 (Figure 1, Table 1), suggesting that halogenase specificity in biosynthetic clusters with two halogenases can be predicted.

Although in vitro experiments with GPA halogenases have failed to show halogenase activity, experiments with chemically halogenated amino acids and peptides have had success. Chemical synthesis of a chlorinated linear hexapeptide bound to a PCP domain was severely inhibited in OxyB crosslinking activity when compared with the nonchlorinated peptide, ${ }^{57}$ implying that chlorination occurs after the first crosslinking. ${ }^{57}$ On the other hand, halogenases appear to be more relaxed in their substrate specificity as culture supernatants of an A. balhimycina bpsC mutant lacking the domain responsible for condensation of the hexapeptide with the last amino acid was able to produce many different halogenated species: linear hexa to dipeptides and a monocyclic hexapeptide. ${ }^{58}$

\section{SULFATION}

Sulfation is less frequently found on naturally occurring GPAs when compared with other modifications such as glycosylation or chlorination, although the number of GPA Stfs characterized has recently grown rapidly. Six have been characterized thus far: TEG12, TEG13, TEG14, Stf AZ205 $_{1}$, StaL and Stf auk $^{14,15,59}$ TEG12, TEG13, TEG14 and Stf $_{\mathrm{AZZ205}}$ are associated with GPA clusters discovered by screening environmental metagenomic libraries. Sulfation by TEG13 and Stf $_{\text {AZ205 }}$ occurs at Bht6, whereas TEG14 sulfates Hpg4, the amino acid commonly glycosylated in other GPA antibiotics. ${ }^{15}$ TEG12 and $\mathrm{Stf}_{\text {auk }}$ act on Dpg3, ${ }^{14,15}$ whereas StaL is specific for Hpg1. ${ }^{59}$ In vitro reactions with overexpressed $\mathrm{Stf}$ and the sulfate donor $3^{\prime}$-phosphoadenosine $5^{\prime}$-phosphosulfate in combination with heterologous expression of Stf genes in the A47934 producer S. toyocaensis have been used to confirm the regiospecifictity of the various Stfs. ${ }^{15,59}$ Validation of regiospecifity was especially important in cases where the Stf was isolated from soil metagenomic samples as the product of the biosynthetic cluster cannot be isolated.

Crystal structures with a Stf alone; in a binary complex with the cofactor product $3^{\prime}$-phosphoadenosine $5^{\prime}$-phosphate (PAP) or the GPA substrate; or in a ternary complex with all three components have been published. The crystal structure of the desulfoA47934:StaL:PAP complex shows that when StaL is bound to its natural substrate desulfo-A47934, the hydroxyl group of Hpg1 is directed toward PAP and is hydrogen bonded to the catalytic His67. ${ }^{60}$ Interestingly, despite the rigidity imposed by oxidative crosslinking of the heptapeptide, desulfo-A47934 showed substantial flexibility. The authors suggest that substrate flexibility may be important for GPA conformational adaptation, allowing GPAs to fit various binding environments such as those present in different tailoring enzymes. ${ }^{60}$ Structures of apo TEG14, ${ }^{61}$ apo TEG12, TEG12 in binary and ternary complexes with teicoplanin aglycone and PAP have also been published but do not shed light on GPA recognition as the GPA is bound outside of the TEG12 active site in the ternary complex. ${ }^{60,62}$

\section{METHYLATION}

Enzymes of GPA clusters have been shown to have $C$-, $O$ - and $\mathrm{N}$-methyltransferase activity. As mentioned previously, during vancosamine biosynthesis EvaC will C-3 methylate its sugar substrate. ${ }^{50}$ $\mathrm{O}$-methylation has been observed on the glucose of pekiskomycin. Mono or di $\mathrm{N}$-methylation occurs at the $\mathrm{N}$-terminal amine of the heptapeptide, on the N-terminal Leu of vancomycin-type GPAs or the N-terminal Hpg of A40926 (Figure 1, Table 1).

The MtfA of chloroeremomycin has been studied both in vivo and in vitro using (S)-adenosyl-L-methionine as a methyl donor. ${ }^{63,64}$ In vitro experiments suggested that when compared with the linear 
and aglycone forms of vancomycin and D- or L-Leu, the preferred substrate for $\mathrm{MtfA}$ is demethyl-vancomycin. ${ }^{57}$ However, when the aglycone and the demethyl-vancomycin were present in equimolar amounts, $>95 \%$ of methylation occurred on the aglycone; this led the authors to suggest that $\mathrm{N}$-methylation occurs after crosslinking but before glycosylation. ${ }^{57} \mathrm{~A}$ crystal structure of $\mathrm{MtfA}_{\text {cep }}$ in complex with (S)-adenosyl-L-methionine modeled with demethyl-vancomycin aglycone suggested that His228 is likely a general base required for methyl transfer. ${ }^{63}$ Site-directed mutagenesis of His 228 resulted in loss of methyltransferase activity, validating the critical role of His $228 .{ }^{63}$ $\mathrm{MtfA}_{\text {cep }}$ also shows substrate flexibility. Heterologous expression of the $\mathrm{MtfA}_{\text {cep }}$ in S. toyocaensis $\Delta$ staL (the desulfo-A47934 producer) led to isolation of methyl-desulfo-A47934, demonstrating that it is able to methylate vancomycin- and teicoplanin-type scaffolds. ${ }^{63}$

Although monomethylation is a typical modification, pekiskomycin, a GPA with an unusual heptapeptide sequence, is dimethylated at the N-terminal Ala (Figure 4e). Banik and Brady ${ }^{16}$ proposed that VEG, a GPA with a predicted structure based on cluster annotation, is dimethylated as the VEG biosynthetic cluster has two N-MtfAs. However, it is unclear whether N-Mtfs only have monomethyl activity. $\mathrm{MtfA}_{\text {cep }}$ shows an ability to dimethylate in vitro ${ }^{64}$ and pekiskomycin is dimethlyated but encodes only one predicted $\mathrm{N}-\mathrm{Mtf}$ (pek30) in its biosynthetic cluster. ${ }^{13}$

\section{TIMING OF BIOSYNTHETIC EVENTS}

Biosynthesis begins with formation of the nascent peptide chain at the NRPS. With the exception of Bht biosynthesis in teicoplanin-type producers, ${ }^{44}$ the nonproteinogenic amino acids and usual sugars are synthesized before or 'just in time' for addition to the GPA scaffold. Oxidative crosslinking then occurs as the peptide is elongated. In vancomycin-type producers, the order of oxidation is $\mathrm{OxyB}>$ OxyA $>$ OxyC. ${ }^{27}$ In teicoplanin-type producers the preferred order of crosslinking follows $\mathrm{OxyB}>\mathrm{OxyE}>\mathrm{OxyA}>\mathrm{OxyC} .{ }^{28}$ Halogenation most likely proceeds after the first crosslinking by $\mathrm{OxyB}^{57}$ but before release of the heptapeptide from the NRPS (further in vitro study with the halogenases is required for clarification). N-methylation occurs after crosslinking but before glycosylation. ${ }^{57}$ In teicoplain and chloroeremomycin, $\mathrm{tGtfB}$ acts first on the aglycone glycosylating Hpg 4. ${ }^{47,53}$ tGtfA then preferentially adds another sugar to Bht6 of a scaffold monoglycosylated at $\mathrm{Hpg} 4 .{ }^{45,51}$ If acylation occurs, it will do so indiscriminately, before or after glycosylation by tGtfA. ${ }^{53} \mathrm{GtfC}$ will then add the secondary sugar resulting in a disaccharide modification at $\mathrm{Hpg} 4 .^{47}$ Because the dominant fermentation product of a Stfdeficient mutant is the fully tailored crosslinked GPA without a sulfate, sulfation is likely to be one of the last modifications that occurs on the heptapeptide scaffold. ${ }^{59}$ However, it is unclear if sulfation occurs before or after glycosylation as most Stf studies have been conducted on the aglycone A47934. ${ }^{59}$

\section{RESISTANCE}

The unique mode of action of GPAs provided hope that the development of pathogen resistance would be slow. Indeed, it took over three decades of use before vancomycin resistance was reported in the clinic. This delay at least in part may be attributed to its relatively low clinical use before the increase of methicillin-resistant S. aureus cases in the early 1980s. ${ }^{2}$ Interestingly, pathogens have employed similar resistance tactics as those developed by the producers to avoid the lethal effects of their own molecules. The detailed mechanism of GPA resistance in VRE was deciphered by the coordinated efforts of the Courvalin and Walsh groups during the 1990s. This review is limited to GPA resistance in actinomycetes. Some excellent reviews can be found on GPA resistance determinants in pathogens. ${ }^{51,65}$

Resistance is an essential prerequisite for the producer to ensure its survival from the lethal effects of its own product. ${ }^{66}$ Producers and other resistant organisms alter the target pentapeptide in Lipid II to terminate in D-Ala-D-Lactate (D-Lac) rather than the canonical D-AlaD-Ala (Figure 2). The reprogramming converts an amide bond into an ester, leaving one less hydrogen bond with the GPA. It also creates a ground state repulsion of the oxygen lone pair in the drug-Lipid II complex. This subtle change dramatically reduces the affinity of GPAs to the modified substrate by 1000 -fold. ${ }^{67,68}$

\section{RESISTANCE APPARATUSES IN ACTINOMYCETES}

The GPA resistance machinery comprises core resistance genes considered essential for resistance, a two component regulatory system, and some accessory genes that either support or enhance resistance in some producers (Figure 6). These resistance elements are

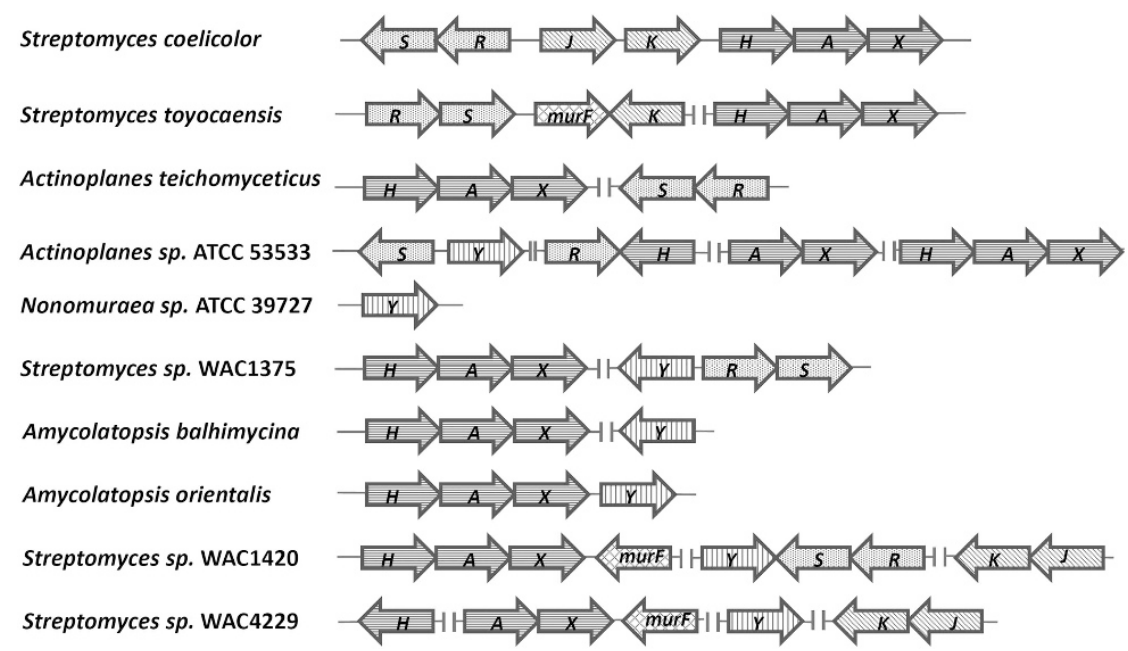

Figure 6 Organization of resistance-associated genes in glycopeptide antibiotic (GPA) producers and the model actinomycete. All genes have been named according to Streptomyces coelicolor nomenclature. Gene orientations are shown with respect to the direction of vanHAX in the respective strain. Genes that are not present in contiguous regions have been marked by two parallel vertical lines. 
not necessarily clustered together or even within their GPA biosynthetic pathways.

\section{CORE RESISTANCE GENES}

Thus far, the core resistance genes vanHAX have been detected in all the published GPA producers except the A40926 producer, which employs an alternative mechanism of self-protection. Frequently, vanHAX is part of the biosynthetic cluster although a draft genome sequence of the balhimycin producer revealed vanHAX $2 \mathrm{Mb}$ away from the cluster. ${ }^{69}$ Irrespective of their location in the genome, coordinated action of these three enzymes modifies the target and imparts resistance. VanH is a D-Lac dehydrogenase that reduces pyruvate to generate the necessary pool of D-Lac precursors. VanA is a D-Ala-D-Lac ligase, a modified form of the traditional D-Ala-D-Ala ligase (Ddl), with a preference for D-Lac that forms the depsipeptide. VanX is a dipeptidase that selectively cleaves D-Ala-D-Ala but does not recognize the modified depsipeptide as a substrate. This targeted action prevents the dipeptide from being loaded to form the canonical pentapeptide stem (Figure 2). These genes have been well studied in the model actinomycete Streptomyces coelicolor, which is not a GPA producer but possesses functional GPA resistance genes.

\section{REGULATORY GENES}

The expression of the vanHAX is typically under the control of a sensory membrane-bound His-kinase (VanS) and its cognate response regulator (VanR). Indirect evidence had suggested that either the GPA or the cell wall intermediates are perceived as a signal by VanS. ${ }^{70,71}$ Recently, a vancomycin photoaffinity probe was synthesized by our group who provided direct evidence for vancomycin binding to VanS S.coelicolor $^{71}$ In 2013, in vivo studies in S. coelicolor by Kwun et al. ${ }^{72}$ indicated that VanS recognizes the complex of pentapeptide bound with vancomycin. The signal perceived by VanS is transferred through its cytoplasmic domain, resulting in His autophosphorylation followed by phosphorylation of the DNA binding response regulator VanR on an Asp residue. The phospho-VanR binds to the region upstream of vanHAX cassette and activates its transcription. ${ }^{73}$ Thus, even though the strains possess the resistance cassette, it is the ability of VanS to sense a GPA that dictates the resistance profile of the strain. For example, acylated ${ }^{51}$ and sulfated ${ }^{74}$ GPAs are not sensed by some types of VanS.

Some producers have bypassed VanRS regulation and constitutively express the resistance genes. For example, the balhimycin producer lacks vanRS. The vanHAX located $2 \mathrm{Mb}$ away from the bal cluster is constitutively expressed as demonstrated by quantitative PCR analysis and liquid chromatography-mass spectrometry of modified pentapeptide in the peptidoglycan of cultures. ${ }^{69}$ Alternatively, studies with A. teichomyceticus showed a decoupled regulatory system ascribed to mutations in vanS. Here the sensor kinase has naturally acquired two point mutations in residues crucial for phosphorylase activity, L215N and G271R, resulting in constitutive VanR activity. ${ }^{75}$

Interestingly, in our recent work we have identified two distinct Streptomyces sp. strains (WAC1420 and WAC4229) that produce pekiskomycin. Their draft genomes show identical biosynthetic cluster organization except for recombinations in WAC4229 resulting in loss of vanRS. ${ }^{13}$ In the same study, we have also identified a GPA producer that lacks the two component regulatory system. ${ }^{13}$ Thus, with the discovery of more biosynthetic pathways, it appears that among GPA producers, the absence of a two component regulatory system is becoming less uncommon.

\section{ACCESSORY RESISTANCE GENES}

The most prevalent accessory gene is $\operatorname{van} Y$ that encodes a $\mathrm{Zn}^{+2}$ dependent D,D-carboxypeptidase that cleaves the C-terminal D-Ala from the pentapeptide, leaving a tetrapeptide that is not recognized by GPAs. ${ }^{76}$ It was first characterized in enterococci where it was shown to enhance the resistance imparted by vanHAX but could not confer high-level resistance alone. ${ }^{76}$ In Nonomuraea sp., VanY has been shown to be the sole resistance determinant. Peptidoglycan analysis of this A40926 producer showed predominantly tetrapeptides confirming carboxypeptidase activity. ${ }^{77}$ A. balhimycina also possess a functional copy of $\operatorname{van} Y$ and the heterologous expression of $\operatorname{van} Y$ in S. coelicolor and its $\triangle$ vanHAX mutant showed a phenotype resembling enterococci. ${ }^{69}$ Similar experiments of expressing a vanY homolog from the UK-68597 biosynthetic cluster in our lab ${ }^{14}$ showed no noticeable phenotypic enhancement of resistance.

Other accessory elements include VanK, VanJ, MurF2 and VanZ. VanK is a homolog of the FemX protein family that is involved in transpeptidation. In S. coelicolor, the housekeeping femX gene cannot recognize the remodeled pentapeptide chain terminating in D-Ala-DLac, and VanK subsequently takes over the role of the endogenous FemX. ${ }^{78}$ VanJ in S. coelicolor ${ }^{79}$ and MurF2 in A. teichomyceticus ${ }^{80}$ have also been implicated in GPA resistance. Finally, homologs of VanZ (confers teicoplanin resistance in enterococci) have been identified in actinomycetes but none so far have been characterized.

\section{CONCLUSION}

The characterization and discovery of GPA biosynthetic enzymes and clusters represent an enormous body of work that has been a driving force in our understanding of NRPS and natural product biosynthesis as well as actinomycete secondary metabolism. Excellent work using a plethora of microbiological, chemical, biochemical, genetic, genomic and metagenomic techniques has enabled this expansion of knowledge. We now know the function of most of the enzymes involved in GPA and LPGA biosynthesis. This information is being harnessed to expand chemical diversity, increasing the pool of bioactive compounds from which therapeutic molecules can be obtained and potentially extending the clinical longevity of this important class of antibiotic. ${ }^{81}$

\section{ACKNOWLEDGEMENTS}

We acknowledge the work of Christopher Walsh has been dominant in our understanding of the biosynthesis of GPAs and resistance in VRE. His leadership and scientific insights have left an indelible stamp on the field that serves to inspire and fuel ongoing efforts to explore GPA chemical biology and drug discovery. Work on GPA's in the authors laboratory is funded by the Canadian Institutes for Health Research (grant no. MT-14981) and the Canada Research Chairs Program. GY is funded by a M.G. DeGroote and CIHR Postdoctoral Fellowships. We also acknowledge Aleksandra Dabkowska for her contribution of fluorescent microscopy images.

1 Levine, D. P. Vancomycin: a history. Clin. Infect. Dis. 42 (Suppl 1), S5-12 (2006).

2 Kirst, H. A., Thompson, D. G. \& Nicas, T. I. Historical yearly usage of vancomycin. Antimicrob. Agents Chemother. 42, 1303-1304 (1998).

3 Williams, A. H. \& Gruneberg, R. N. Teicoplanin. J. Antimicrob. Chemother. 14, 441-445 (1984).

4 Cynamon, M. H. \& Granato, P. A. Comparison of the in vitro activities of teichomycin A2 and vancomycin against staphylococci and enterococci. Antimicrob. Agents Chemother. 21, 504-505 (1982).

5 Pelzer, S. et al. Identification and analysis of the balhimycin biosynthetic gene cluster and its use for manipulating glycopeptide biosynthesis in Amycolatopsis mediterranei DSM5908. Antimicrob. Agents Chemother. 43, 1565-1573 (1999).

6 van Wageningen, A. M. et al. Sequencing and analysis of genes involved in the biosynthesis of a vancomycin group antibiotic. Chem. Biol. 5, 155-162 (1998). 
7 Woertz, T., Dietz, S., Zerbe, K. \& Robinson, J. A. Amycolatopsis orientalis vancomycin biosynthesis cluster, strain ATCC19795. Accession no. HE589771. GenBank (2011).

8 Jeong, $\mathrm{H}$. et al. Genome sequence of the vancomycin-producing Amycolatopsis orientalis subsp. orientalis strain KCTC 9412T. Genome Announc. 1, e00408-13 (2013).

9 Sosio, M., Stinchi, S., Beltrametti, F., Lazzarini, A. \& Donadio, S. The gene cluster for the biosynthesis of the glycopeptide antibiotic A40926 by nonomuraea species. Chem. Biol. 10, 541-549 (2003).

10 Pootoolal, J. et al. Assembling the glycopeptide antibiotic scaffold: the biosynthesis of A47934 from Streptomyces toyocaensis NRRL15009. Proc. Natl Acad. Sci. USA 99, 8962-8967 (2002)

11 Sosio, M. et al. Organization of the teicoplanin gene cluster in Actinoplanes teichomyceticus. Microbiology 150 (Pt 1), 95-102 (2004).

$12 \mathrm{Li}, \mathrm{T}$. L. et al. Biosynthetic gene cluster of the glycopeptide antibiotic teicoplanin: characterization of two glycosyltransferases and the key acyltransferase. Chem. Biol. 11, 107-119 (2004)

13 Thaker, M. N. et al. Identifying producers of antibacterial compounds by screening for antibiotic resistance. Nat. Biotechnol. 31, 922-927 (2013).

14 Yim, G. et al. Characterization of the UK-68,597 biosynthetic cluster: harnessing the synthetic capabilities of glycopeptide tailoring enzymes Unpublished.

15 Banik, J. J., Craig, J. W., Calle, P. Y. \& Brady, S. F. Tailoring enzyme-rich environmenta DNA clones: a source of enzymes for generating libraries of unnatural natural products. J. Am. Chem. Soc. 132, 15661-15670 (2010)

16 Banik, J. J. \& Brady, S. F. Cloning and characterization of new glycopeptide gene clusters found in an environmental DNA megalibrary. Proc. Natl Acad. Sci. USA 105, 17273-17277 (2008).

17 Owen, J. G. et al. Mapping gene clusters within arrayed metagenomic libraries to expand the structural diversity of biomedically relevant natural products. Proc. Nat Acad. Sci. USA 110, 11797-11802 (2013)

18 Marahiel, M. A. \& Essen, L. O. Chapter 13Nonribosomal peptide synthetases mechanistic and structural aspects of essential domains. Methods Enzymol. 458, 337-351 (2009)

19 Hahn, M. \& Stachelhaus, T. Harnessing the potential of communication-mediating domains for the biocombinatorial synthesis of nonribosomal peptides. Proc. Natl Acad. Sci. USA 103, 275-280 (2006).

20 Stachelhaus, T., Mootz, H. D. \& Marahiel, M. A. The specificity-conferring code of adenylation domains in nonribosomal peptide synthetases. Chem. Biol. 6, 493-505 (1999).

21 Linne, U., Doekel, S. \& Marahiel, M. A. Portability of epimerization domain and role of peptidyl carrier protein on epimerization activity in nonribosomal peptide synthetases. Biochemistry 40, 15824-15834 (2001).

22 Stachelhaus, T. \& Walsh, C. T. Mutational analysis of the epimerization domain in the initiation module PheATE of gramicidin S synthetase. Biochemistry 39, 5775-5787 (2000).

23 Luo, L. et al. Timing of epimerization and condensation reactions in nonribosoma peptide assembly lines: kinetic analysis of phenylalanine activating elongation modules of tyrocidine synthetase B. Biochemistry 41, 9184-9196 (2002).

24 Medema, M. H. et al. antiSMASH: rapid identification, annotation and analysis of secondary metabolite biosynthesis gene clusters in bacterial and fungal genome sequences. Nucleic Acids Res. 39 (Web Server issue), W339-W346 (2011).

25 Pootoolal, J., Neu, J. \& Wright, G. D. Glycopeptide antibiotic resistance. Annu. Rev. Pharmacol. Toxicol. 42, 381-408 (2002).

26 Nicolaou, K. C., Boddy, C. N., Brase, S. \& Winssinger, N. Chemistry, biology, and medicine of the glycopeptide antibiotics. Angew. Chem. Int. Ed. Engl. 38, 2096-2152 (1999).

27 Stegmann, E. et al. Genetic analysis of the balhimycin (vancomycin-type) oxygenase genes. J. Biotechnol. 124, 640-653 (2006)

28 Hadatsch, B. et al. The biosynthesis of teicoplanin-type glycopeptide antibiotics: assignment of p450 mono-oxygenases to side chain cyclizations of glycopeptide a47934. Chem. Biol. 14, 1078-1089 (2007).

29 Cryle, M. J., Staaden, J. \& Schlichting, I. Structural characterization of CYP165D3, a cytochrome P450 involved in phenolic coupling in teicoplanin biosynthesis. Arch. Biochem. Biophys. 507, 163-173 (2011)

30 Li, Z., Rupasinghe, S. G., Schuler, M. A. \& Nair, S. K. Crystal structure of a phenolcoupling P450 monooxygenase involved in teicoplanin biosynthesis. Proteins 79, 1728-1738 (2011)

31 Woithe, K. et al. Oxidative phenol coupling reactions catalyzed by OxyB: a cytochrome P450 from the vancomycin producing organism. implications for vancomycin biosynthesis. J. Am. Chem. Soc. 129, 6887-6895 (2007)

32 Chen, H., Tseng, C. C., Hubbard, B. K. \& Walsh, C. T. Glycopeptide antibiotic biosynthesis: enzymatic assembly of the dedicated amino acid monomer (S)-3, 5-dihydroxyphenylglycine. Proc. Natl Acad. Sci. USA 98, 14901-14906 (2001).

33 Pfeifer, V. et al. A polyketide synthase in glycopeptide biosynthesis: the biosynthesis of the non-proteinogenic amino acid (S)-3,5-dihydroxyphenylglycine. J. Biol. Chem. 276, 38370-38377 (2001)

34 Sandercock, A. M. et al. Biosynthesis of the di-hydroxyphenylglycine constituent of the vancomycin-group antibiotic chloroeremomycin. Chem. Commun. 14, 1252-1253 (2001).

35 Hubbard, B. K., Thomas, M. G. \& Walsh, C. T. Biosynthesis of L-p-hydroxyphenylglycine, a non-proteinogenic amino acid constituent of peptide antibiotics. Chem. Biol. 7 931-942 (2000)
36 Chen, H. \& Walsh, C. T. Coumarin formation in novobiocin biosynthesis: betahydroxylation of the aminoacyl enzyme tyrosyl-S-NovH by a cytochrome P450 Novl. Chem. Biol. 8, 301-312 (2001).

37 Chen, H., Hubbard, B. K., O'Connor, S. E. \& Walsh, C. T. Formation of beta-hydroxy histidine in the biosynthesis of nikkomycin antibiotics. Chem. Biol. 9, 103-112 (2002)

38 Donadio, S., Sosio, M., Stegmann, E., Weber, T. \& Wohlleben, W. Comparative analysis and insights into the evolution of gene clusters for glycopeptide antibiotic biosynthesis. Mol. Genet. Genomics 274, 40-50 (2005).

39 Puk, 0. et al. Biosynthesis of chloro-beta-hydroxytyrosine, a nonproteinogenic amino acid of the peptidic backbone of glycopeptide antibiotics. J. Bacteriol. 186, 6093-6100 (2004)

40 Recktenwald, J. et al. Nonribosomal biosynthesis of vancomycin-type antibiotics: a heptapeptide backbone and eight peptide synthetase modules. Microbiology 148 (Pt 4), 1105-1118 (2002).

41 Puk, O. et al. Glycopeptide biosynthesis in Amycolatopsis mediterranei DSM5908 function of a halogenase and a haloperoxidase/perhydrolase. Chem. Biol. 9, 225-235 (2002)

42 Cryle, M. J., Meinhart, A. \& Schlichting, I. Structural characterization of OxyD, a cytochrome P450 involved in beta-hydroxytyrosine formation in vancomycin biosynthesis. J. Biol. Chem. 285, 24562-24574 (2010).

43 Mulyani, S. et al. The thioesterase Bhp is involved in the formation of betahydroxytyrosine during balhimycin biosynthesis in Amycolatopsis balhimycina. Chembiochem. 11, 266-271 (2010).

44 Stinchi, S. et al. A derivative of the glycopeptide A40926 produced by inactivation of the beta-hydroxylase gene in Nonomuraea sp. ATCC39727. FEMS Microbiol. Lett. 256, 229-235 (2006).

45 Truman, A. W. et al. Chimeric glycosyltransferases for the generation of hybrid glycopeptides. Chem. Biol. 16, 676-685 (2009).

46 Losey, H. C. et al. Incorporation of glucose analogs by GtfE and GtfD from the vancomycin biosynthetic pathway to generate variant glycopeptides. Chem. Biol. 9, 1305-1314 (2002)

$47 \mathrm{Lu}, \mathrm{W}$. et al. Characterization of a regiospecific epivancosaminyl transferase GtfA and enzymatic reconstitution of the antibiotic chloroeremomycin. Proc. Natl Acad. Sci. USA 101, 4390-4395 (2004).

48 Losey, H. C. et al. Tandem action of glycosyltransferases in the maturation of vancomycin and teicoplanin aglycones: novel glycopeptides. Biochemistry 40, 4745-4755 (2001)

49 Mulichak, A. M. et al. Structure of the TDP-epi-vancosaminyltransferase GtfA from the chloroeremomycin biosynthetic pathway. Proc. Natl Acad. Sci. USA 100, 9238-9243 (2003)

$50 \mathrm{Chen}, \mathrm{H}$. et al. Deoxysugars in glycopeptide antibiotics: enzymatic synthesis of TDP-Lepivancosamine in chloroeremomycin biosynthesis. Proc. Natl Acad. Sci. USA 97, 11942-11947 (2000)

51 Kahne, D., Leimkuhler, C., Lu, W. \& Walsh, C. Glycopeptide and lipoglycopeptide antibiotics. Chem. Rev. 105, 425-448 (2005).

52 Kruger, R. G. et al. Tailoring of glycopeptide scaffolds by the acyltransferases from the teicoplanin and A-40,926 biosynthetic operons. Chem. Biol. 12, 131-140 (2005).

53 Howard-Jones, A. R. et al. Kinetic analysis of teicoplanin glycosyltransferases and acyltransferase reveal ordered tailoring of aglycone scaffold to reconstitute mature teicoplanin. J. Am. Chem. Soc. 129, 10082-10083 (2007)

$54 \mathrm{Ho}$, J. Y. et al. Glycopeptide biosynthesis: Dbv21/Orf2 from dbv/tcp gene clusters are $\mathrm{N}-$ Ac-Glm teicoplanin pseudoaglycone deacetylases and Orf15 from cep gene cluster is a Glc-1-P thymidyltransferase. J. Am. Chem. Soc. 128, 13694-13695 (2006).

55 Gerhard, U., Mackay, J. P., Maplestone, R. A. \& Williams, D. H. The role of the sugar and chlorine substituents in the dimerization of vancomycin antibiotics. J. Am. Chem. Soc. 115, 232-237 (1993).

56 Williams, D. H. \& Bardsley, B. The vancomycin group of antibiotics and the fight against resistant bacteria. Ange. Chem. Int. Ed. 38, 1172-1193 (1999).

57 Schmartz, P. C. et al. Substituent effects on the phenol coupling reaction catalyzed by the vancomycin biosynthetic P450 enzyme OxyB. Angew. Chem. Int. Ed. Engl. 51, 11468-11472 (2012).

58 Bischoff, D. et al. The biosynthesis of vancomycin-type glycopeptide antibiotics-a model for oxidative side-chain cross-linking by oxygenases coupled to the action of peptide synthetases. Chembiochem. 6, 267-272 (2005).

59 Lamb, S. S., Patel, T., Koteva, K. P. \& Wright, G. D. Biosynthesis of sulfated glycopeptide antibiotics by using the sulfotransferase StaL. Chem. Biol. 13, 171-181 (2006).

60 Shi, R. et al. Sulfonation of glycopeptide antibiotics by sulfotransferase StaL depends on conformational flexibility of aglycone scaffold. Proc. Natl Acad. Sci. USA 109 , 11824-11829 (2012)

61 Bick, M. J., Banik, J. J., Darst, S. A. \& Brady, S. F. The 2.7 A resolution structure of the glycopeptide sulfotransferase Teg14. Acta. Crystallogr. D Biol. Crystallogr. 66 (Pt 12), 1278-1286 (2010)

62 Bick, M. J., Banik, J. J., Darst, S. A. \& Brady, S. F. Crystal structures of the glycopeptide sulfotransferase Teg12 in a complex with the teicoplanin aglycone. Biochemistry 49, 4159-4168 (2010).

$63 \mathrm{Shi}, \mathrm{R}$. et al. Structure and function of the glycopeptide N-methyltransferase MtfA, a tool for the biosynthesis of modified glycopeptide antibiotics. Chem. Biol. 16, 401-410 (2009).

64 O'Brien, D. P. et al. Expression and assay of an -methyltransferase involved in the biosynthesis of a vancomycin group antibiotic. Chem. Commun. 1, 103-104 (2000).

65 Courvalin, P. Vancomycin resistance in gram-positive cocci. Clin. Infect. Dis. 42 (Suppl 1), S25-S34 (2006). 
66 Cundliffe, E. \& Demain, A. L. Avoidance of suicide in antibiotic-producing microbes. J. Ind. Microbiol. Biotechnol. 37, 643-672 (2010).

67 McComas, C. C., Crowley, B. M. \& Boger, D. L. Partitioning the loss in vancomycin binding affinity for D-Ala-D-Lac into lost $\mathrm{H}$-bond and repulsive lone pair contributions. J. Am. Chem. Soc. 125, 9314-9315 (2003).

68 Bugg, T. D. et al. Molecular basis for vancomycin resistance in Enterococcus faecium BM4147: biosynthesis of a depsipeptide peptidoglycan precursor by vancomycin resistance proteins $\mathrm{VanH}$ and VanA. Biochemistry 30, 10408-10415 (1991).

69 Schaberle, T. F. et al. Self-resistance and cell wall composition in the glycopeptide producer Amycolatopsis balhimycina. Antimicrob. Agents Chemother. 55, 4283-4289 (2011).

70 Baptista, M., Depardieu, F., Courvalin, P. \& Arthur, M. Specificity of induction of glycopeptide resistance genes in Enterococcus faecalis. Antimicrob. Agents Chemother. 40, 2291-2295 (1996).

71 Koteva, K. et al. A vancomycin photoprobe identifies the histidine kinase VanSsc as a vancomycin receptor. Nat. Chem. Biol. 6, 327-329 (2010).

72 Kwun, M. J., Novotna, G., Hesketh, A. R., Hill, L. \& Hong, H. J. In vivo studies suggest that induction of VanS-dependent vancomycin resistance requires binding of the drug to D-Ala-D-Ala termini in the peptidoglycan cell wall. Antimicrob. Agents Chemother 57, 4470-4480 (2013).

73 Arthur, M., Molinas, C. \& Courvalin, P. The VanS-VanR two-component regulatory system controls synthesis of depsipeptide peptidoglycan precursors in Enterococcus faecium BM4147. J. Bacteriol. 174, 2582-2591 (1992).
74 Kalan, L., Perry, J., Koteva, K., Thaker, M. \& Wright, G. Glycopeptide sulfation evades resistance. J. Bacteriol. 195, 167-171 (2013).

75 Beltrametti, F. et al. Resistance to glycopeptide antibiotics in the teicoplanin producer is mediated by van gene homologue expression directing the synthesis of a modified cell wall peptidoglycan. Antimicrob. Agents Chemother. 51, 1135-1141 (2007).

76 Wright, G. D., Molinas, C., Arthur, M., Courvalin, P. \& Walsh, C. T. Characterization of vanY, a DD-carboxypeptidase from vancomycin-resistant Enterococcus faecium BM4147. Antimicrob. Agents Chemother. 36, 1514-1518 (1992).

77 Marcone, G. L. et al. Novel mechanism of glycopeptide resistance in the A40926 producer Nonomuraea sp. ATCC 39727. Antimicrob. Agents Chemother. 54, 2465-2472 (2010).

78 Hong, H. J. et al. Characterization of an inducible vancomycin resistance system in Streptomyces coelicolor reveals a novel gene (vanK) required for drug resistance. Mol. Microbiol. 52, 1107-1121 (2004).

79 Novotna, G., Hill, C., Vincent, K., Liu, C. \& Hong, H. J. A novel membrane protein, VanJ, conferring resistance to teicoplanin. Antimicrob. Agents Chemother. 56, 1784-1796 (2012).

80 Serina, S., Radice, F., Maffioli, S., Donadio, S. \& Sosio, M. Glycopeptide resistance determinants from the teicoplanin producer Actinoplanes teichomyceticus. FEMS Microbiol. Lett. 240, 69-74 (2004).

81 Thaker, M. N. \& Wright, G. D. Opportunities for synthetic biology in antibiotics: expanding glycopeptide chemical diversity. ACS Synth. Biol. (e-pub ahead of print 28 December 2013; doi:10.1021/sb300092n). 